\title{
Nail Involvement in Psoriatic Patients and Association with Onychomycosis: Results from a Cross-Sectional Study Performed in a Military Hospital in Tunisia
}

\author{
Fatma Jendoubi $^{a} \quad$ Imene Ben Lagha ${ }^{a}$ Faten Rabhi ${ }^{a} \quad$ Nejib Doss $^{a} \quad$ Ali Mrabet $^{b}$ \\ Kahena Jaber ${ }^{a}$ Mohamed Raouf Dhaoui ${ }^{a}$ \\ a Dermatology Department, Military Hospital of Tunis, Tunis, Tunisia; ${ }^{b}$ Public Health and Epidemiology Department, \\ Faculty of Medicine of Tunis, University of Tunis El Manar, Tunis, Tunisia
}

\author{
Keywords \\ Nail disorder · Nail inflammatory disease · Nail psoriasis · \\ Psoriasis · Nail fungus
}

\begin{abstract}
Nail psoriasis has variable prevalence and heterogeneous aspects. Many of them could mimic onychomycosis (OM). It has been suggested that patients with nail psoriasis are at high risk of OM. The aim of our study was to determine the epidemiological and clinical characteristics of nail psoriasis and to estimate the frequency and the factors associated with $\mathrm{OM}$ in psoriatic patients. The studied group included 163 patients with psoriasis aged 18 years or older. Epidemiological and clinical data, as well as the severity of skin and nails disease by evaluating the Psoriasis Area Severity Index (PASI) and Nail Area Psoriasis Severity Index (NAPSI) scores were specified. Mycological testing was performed for patients with nail alterations. Nail involvement was found in $71.2 \%$ of patients. The most common nail alterations were subungual hyperkeratosis and onycholysis. The mean NAPSI score was 11.6. Mycological testing was performed in 104 patients with onychodystrophy. OM was diagnosed in 53\% of the cases. Dermatophytes were the most isolated patho-
\end{abstract}

gens. OM was associated with male gender, but not with age, NAPSI, or PASI score. Psoriasis is one of the dermatoses that most commonly affect the nail. Available data about the association between nail psoriasis and $\mathrm{OM}$ are controversial. However, mycological testing should be routinely performed on psoriatic nails.

(c) 2019 S. Karger AG, Basel

\section{Introduction}

Psoriasis is one of the dermatoses that most commonly affects the nails. Over a lifetime, $80-90 \%$ of psoriatic patients develop nail alterations [1]. Nail involvement may have a major functional and psychological impact. It is also considered as an indicator of psoriatic arthritis (PsA) [2]. Psoriatic nail involvement may sometimes mimic onychomycosis $(\mathrm{OM})$. The latter represents the most frequent cause of nail disease, accountable for over $50 \%$ of nail alterations [3]. Sometimes, it may be difficult to clinically differentiate between the two diseases [4].

Fatma Jendoubi and Imene Ben Lagha are first co-authors.

\section{KARGER}

(c) 2019 S. Karger AG, Basel

E-Mail karger@karger.com

www.karger.com/sad
Imene Ben Lagha

Dermatology Department

Military Hospital of Tunis, Montfleury

1008 Tunis (Tunisia)

E-Mail imenebenlagha@gmail.com 
It has been hypothesized that morphological abnormalities in psoriatic nails are predisposing factors for $\mathrm{OM}$ and that prevalence of $\mathrm{OM}$ in patients with nail psoriasis might be higher than in the general population. Immunosuppressive therapies in psoriasis might also play a role in developing OM. On the other hand, the higher immune response against microbial skin infections in psoriatic patients as well as the fast turnover of psoriatic nails may be considered as an effective defense against fungus.

Considering that OM can aggravate nail abnormalities in psoriasis by koebnerization, it may be useful to differentiate between psoriatic nail alterations and an underlying $\mathrm{OM}$ in psoriatic patients. Documented prevalence of $\mathrm{OM}$ in patients with nail psoriasis varies considerably and conclusions concerning the prevalence and vulnerability of psoriatic nails for OM are ambiguous. Thus, we aimed to characterize nail abnormalities in patients with psoriasis and to assess the prevalence of OM in the Dermatology Department of the Military Hospital of Tunis. Our findings in this specific population were then compared with the corresponding available data in the literature.

\section{Materials and Methods}

A cross-sectional study including patients with psoriasis aged 18 years or older was performed in the Dermatology Department of the Military Hospital of Tunis, Tunisia, between May 2016 and April 2017. Informed consent was obtained from the study participants before enrollment. Good Clinical Practice and the declaration of Helsinki have been adhered to.

For each patient, the following data were recorded: age, gender, family history of OM, and personal predisposing factors for OM (venous or arterial insufficiency, diabetes, hyperhidrosis, and lower limb trauma). Duration of the disease, diagnosed PsA or PsArelated symptoms (history of joint, spine, or entheseal inflammatory arthritis), and psoriasis treatment were also specified for all patients.

Dermatological examination specified the clinical form of psoriasis, nail alterations type, and number of fingernails and toenails involved, the Psoriasis Area and Severity Index (PASI) score assessing the severity of skin involvement, and the Nail Area Psoriasis Severity Index (NAPSI) score assessing the severity of nail involvement. Consenting patients with nail involvement underwent a mycological examination at the Mycology Department of the Military Hospital of Tunis. Samples of the involved fingernail, toenail, or both were collected.

A nail specimen analysis was performed for each patient using direct microscopy examination with $\mathrm{KOH}$ and culture in two different types of Sabouraud dextrose agar: with chloramphenicol and with chloramphenicol and actidione. The mycological examination was considered as positive if direct examination showed the presence of typical fungal mycelia consistent with the presence of dermatophytes or when the culture was positive and isolated a fungus (Candida and/or dermatophyte).

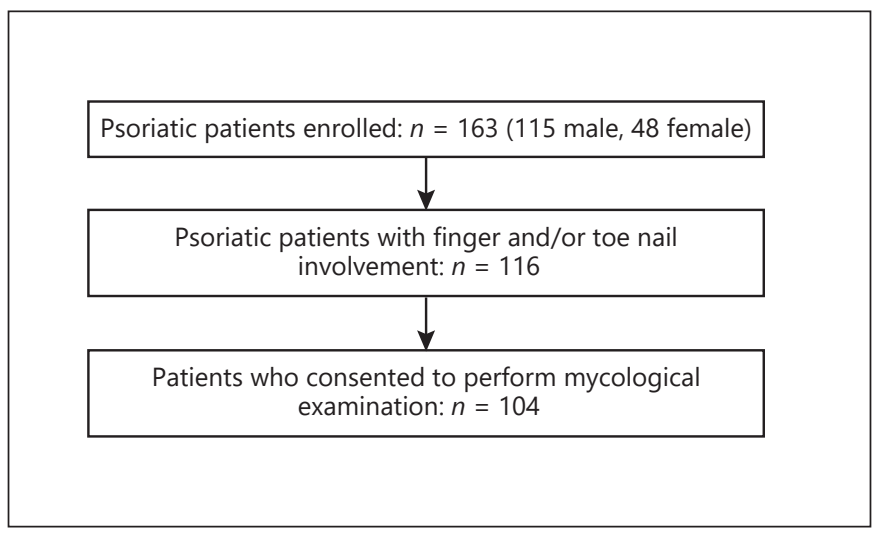

Fig. 1. Patients enrollment for clinical and mycological examination.

Data analysis was performed using SPSS software (version 24, SPSS, Inc., Chicago, IL, USA). Student's $t$ test was used for continuous variables and Pearson's $\chi^{2}$ test or Fisher's exact test for categorical variables. $p$ values $<0.05$ were considered to be statistically significant.

\section{Results}

We enrolled 163 psoriatic patients (115 males, $48 \mathrm{fe}$ males, sex ratio: 2.4 ) with a mean age of 44.2 years (range: 18-80, standard deviation [SD]: 16.16) (Fig. 1). Mean duration of the disease was 10 years (range: $0.16-60$, SD: 11.47). A total of 17 patients (10.4\%) reported a history of $\mathrm{OM}$ in their immediate family circle. Risk factors of OM were reported only in $20 \%$ of patients (diabetes: $11 \%$, hyperhidrosis: $4 \%$, lower limb trauma: $4 \%$, and venous insufficiency: $1 \%)$. Ten patients were diagnosed with PsA (6.1\%), whereas 37 patients reported PsA-related symptoms $(22.7 \%)$. Immunosuppressive therapies consisted in corticosteroids (86.5\%), methotrexate (25.2\%), and etanercept (1 patient). The mean PASI score was 10.9 (range: $0-59$, SD: 12.08$)$. Among our patients, $71.2 \%(n=116)$ had nail involvement of fingers and/or toes. Fingernails were involved in $10.4 \%$ of patients with nail abnormalities, toenails in $36.2 \%$, and both hands and feet in $53.4 \%$. The prevalence of nail involvement was $90 \%$ in patients with PsA. One patient had isolated nail psoriasis.

Involvement of the nail matrix consisted in nail pitting (27.5\% of patients), trachyonychia (26.7\%), and Beau's lines (22.4\%). Involvement of the nail bed consisted in onycholysis $(62.9 \%)$, subungual hyperkeratosis $(65.5 \%)$, salmon patches $(7.7 \%)$, and splinter hemorrhages (8.6\%). 
Table 1. Comparison of nail abnormalities in all psoriatic patients enrolled and in psoriatic patients with onychomycosis

\begin{tabular}{llll}
\hline & $\begin{array}{l}\text { Patients } \\
\text { with nail ab- } \\
\text { normalities } \\
(n=116)\end{array}$ & $\begin{array}{l}\text { Patients with nail } \\
\text { abnormalities and } \\
\text { onychomycosis } \\
(n=55)\end{array}$ & $p$ value \\
& 76 & 44 & 0.066 \\
Subungual hyperkeratosis & 73 & 36 & 0.376 \\
Onycholysis & 32 & 17 & 0.153 \\
Pitting nails & 31 & 16 & 0.474 \\
Leukonychia & 23 & 15 & 0.185 \\
Pachyonychia & 25 & 14 & 0.539 \\
Xanthonychia & 34 & 13 & 0.206 \\
Longitudinal ridges & 24 & 12 & 0.583 \\
Melanonychia & 10 & 8 & 0.06 \\
Splinter hemorrhages & 11 & 7 & 0.055 \\
Trachyonychia & 26 & 9 & 0.499 \\
Beau's lines & 9 & 6 & 0.35 \\
Salmon patches & & & \\
\hline
\end{tabular}

The median NAPSI score was 11.59 (range: 0-106, SD: 19.72) (Table 1).

Nail abnormalities which are not included in the NAPSI score were also observed, such as longitudinal ridges $(29.3 \%)$, xanthonychia $(21.5 \%)$, melanonychia (20.6\%), pachyonychia (19.8\%), and trachyonychia (9.4\%) (Table 1).

PsA was more prevalent in patients with nail involvement ( 7.1 vs. $2.2 \%)$, but the difference was not statistically significant. Patients with nail involvement had a longer duration of the disease (10.9 vs. 7.8 years) and a more severe cutaneous involvement (PASI 11.6 vs. 8.9) than patients without nail involvement but the difference was again not significant. However, patients with nail involvement were older than those without nail involvement $(p<0.05)$, without significant correlation with gender. More interestingly, we found a positive correlation between the severity of nail involvement (assessed by NAPSI score) and patients' age, duration of the disease, and severity of the cutaneous involvement assessed by PASI score $(p<0.05)$. Also, the mean NAPSI score among patients with PsA (16.3) was higher than in patients without PsA (10.22), but the difference was not statistically significant.

Among patients with nail involvement $(n=116), 104$ consented to perform a mycological examination (89\%) (Fig. 1). Specimens were collected from 57 fingernails and 100 toenails. Typical mycelia consisting in the presence of dermatophytes was found in 45 specimens. Cultures isolated fungi in $14 \%$ of fingernails and in $34 \%$ of toenails. The isolated fungal species from fingernails were Candi- da parapsilosis (9\%), Candida albicans (3.5\%), and Candida non-albicans (1.5\%). Trichophyton rubrum was the only specimen isolated in toenails. Thus, $53 \%$ of patients who underwent mycological examination $(n=55)$ were considered as having an OM. OM was more prevalent in male patients $(p<0.005)$.

There were no correlations between the presence of fungal infection and the type of nail alterations. The NAPSI score was slightly higher in patients with OM (16.2) compared to patients without OM (14.7; $p>0.05)$. We did not find a significant correlation between the presence of fungal infection and the presence of the classical risk factors of OM cited before nor the use of immunosuppressive therapies.

\section{Discussion/Conclusion}

Our study has some limitations such as the recruitment of patients in a military population and the absence of a control group. Also, nail involvement was systematically considered as related to psoriasis and fungal infection was considered as a secondary phenomenon.

The prevalence of nail involvement in psoriatic patients was $71.2 \%$. Multiple previous reports found close results with a prevalence varying from 60 to $80 \%$ [5-9]. A few studies reported a lower prevalence from 20 to $40 \%$ [10-12]. In the literature as in the present study, pitting nails was the most frequently encountered nail matrix involvement in psoriasis [5-7, 13, 14]. The most common nail bed involvement were onycholysis and subungual hyperkeratosis as reported in the literature $[7,13]$.

Approximately, $90 \%$ of psoriatic patients develop nail psoriasis during their lifetime [1]. The association of nail alterations with age is controversial. In the present study, older patients were more likely to have nail changes as reported previously $[5,15]$. Moreover, our results showed that age was also related to a more severe nail involvement. Other studies denied this association $[7,16]$. Nail psoriasis is often related to cutaneous psoriasis severity $[12,17]$ and disease duration $[6,10]$. Even if statistically nonsignificant, our results were consistent with this observation. Furthermore, the severity of nail alterations was significantly related to the severity and duration of disease.

The present study showed the highest prevalence of OM in psoriatic patients (53\%) compared with other previous studies outlined in Table 2. The literature data on this issue is contradictory. Some of the studies found a higher OM prevalence in psoriatic patients 
Table 2. Literature review of onychomycosis in psoriatic nails

\begin{tabular}{llll}
\hline First author, country & $\begin{array}{l}\text { Onychomycosis } \\
\text { prevalence in } \\
\text { psoriatics, \% }\end{array}$ & $\begin{array}{l}\text { Onychomycosis } \\
\text { prevalence in } \\
\text { controls, \% }\end{array}$ & Conclusions \\
\hline Szepes [28], Hungary & 63.1 & 66 & No significant difference \\
Staberg [29], Denmark & 26.9 & 19.5 & $\begin{array}{l}\text { No significant difference, high rate of yeasts and dermatophytes } \\
\text { Gignificant difference, high rate of dermatophytes }\end{array}$ \\
Gupta [18], USA & 27 & 6.9 & No significant difference, high rate of yeasts \\
Kiellberg Larsen [21], Denmark & 30.4 & 19.6 & No significant difference, high rate of yeasts and dermatophytes \\
Salomon [5], Poland & 21.5 & 12.7 & High rate of dermatophytes \\
Hamnerius [31], Sweden & 18 & - & No significant difference \\
Kaçar [24], Turkey & 4.6 & 7.4 & $\begin{array}{l}\text { No significant difference, high rate of dermatophytes } \\
\text { Significant difference }\end{array}$ \\
Leibovici [27], Israel & 47.1 & 28.4 & High prevalence of onychomycosis in psoriatics \\
Zisova [25], Bulgaria/Greece & 62 & - & Only nondermatophytic molds and yeast \\
Natarajan [32], India & 47.9 & - & No significant difference, high rate of nondermatophytic molds \\
Romaszkiewicz [26], Poland & 23.5 & High rate of nondermatophytic molds and yeasts \\
Chaowattanapanit [33], Thailand & $27.4-32.3$ & - & High rate of dermatophytes \\
Shemer [12], Israel & 34.3 & - &
\end{tabular}

compared with controls, while others did not report a significant difference between the two groups. A systematic review including some of the previous studies concluded that the overall prevalence of $\mathrm{OM}$ in patients with psoriasis was $18 \%$ [8]. Gupta et al. [18] estimated that similarly to HIV-positive and dialysis patients, psoriatic patients were considered as highly predisposed to develop OM.

The high prevalence in our study may be explained by a recruitment bias: the military population is exposed to multiple predisposing factors for OM such as repeated trauma, occlusive footwear, and communal bathing. The pathogenesis of OM in psoriasis is not well established. Some authors claim that the loss of the compact orthokeratotic layer of the nail plate and the onycholysis seen in psoriatic nails alter the physical protection of the nail against microorganisms [19]. Besides, it has been suggested that the HLA-DR locus may play a role in the genetic susceptibility to OM in nail psoriasis [20]. Immunosuppressive agents administered in psoriasis may also alter the immune status and facilitate fungal infection. However, according to our results, there was no correlation between OM and the use of topical steroids or methotrexate. On the other hand, the rapid growth of the affected nails in psoriasis is thought to decrease the opportunity of fungal invasion of the nail [21]. In addition, antimicrobial peptides like psoriasins are upregulated in psoriasis and should increase the protection against skin infections [22]. Another hypothesis is that OM could trigger nail psoriasis through a koebner phenomenon [23]. In our study, the NAPSI score was higher in patients with con- comitant OM (16.2 vs. 14.4), but the difference was not statistically significant. Similar results were previously reported [24-26].

The most frequent isolated agent in patients with $\mathrm{OM}$ was T. rubrum. In other studies, dermatophytes were also most commonly responsible for $\mathrm{OM}$ in psoriasis $[5,18$, 24]. Only a few studies looked for possible associations of OM with clinical and demographic characteristics of psoriasis patients. A significant association with male gender $(p=0.015)$ was observed in our study and two other studies $[18,27]$. We could speculate that some nail alteration type could be more associated with the presence of concomitant OM. In our study, we did not find any correlation between the nail alteration type and the presence of OM.

In other terms, nail alterations such as pitting, salmon patches, and splinter hemorrhages considered as highly suspicious of nail psoriasis were not correlated with the absence of fungal infection. To our knowledge, no similar data was previously reported in the literature.

Regarding the absence of correlation between the type and the severity of the nail involvement and the presence of a concomitant fungal infection, OM should be, in our opinion, systematically ruled out in psoriatic patients with nail involvement.

In conclusion, nail involvement in psoriatic patients is common. The demographic and pathogenic link with OM remains a matter of debate. However, mycological testing should be routinely performed on psoriatic nails. 


\section{Statement of Ethics}

The study participants have given their written informed consent. The study protocol has been approved by the institute's committee on human research.

\section{Disclosure Statement}

The authors have no conflicts of interest to declare.

\section{Author Contributions}

Fatma Jendoubi: patients' recruitment and follow-up, manuscript writing, and literature review. Imene Ben Lagha: literature review and manuscript writing. Faten Rabhi: patients' recruitment. Nejib Doss: patient's recruitment and revision of the manuscript. Ali Mrabet: statistical analysis. Kahena Jaber: patients' recruitment and revision of the manuscript. Mohamed Raouf Dhaoui: patients' recruitment.

\section{References}

1 Langley RG, Daudén E. Treatment and management of psoriasis with nail involvement: a focus on biologic therapy. Dermatology. 2010;221 Suppl 1:29-42.

2 Jullien D, Fournié B, Boulinguez S. [Nail psoriasis: a marker of profound inflammation]. Ann Dermatol Venereol. 2011 Jun;138(6 Suppl 1):H13-7. French.

3 Williams HC. The epidemiology of onychomycosis in Britain. Br J Dermatol. 1993 Aug; 129(2):101-9.

4 Sánchez-Regaña ML, Videla S, Villoria J, Domingo H, Macaya A, Ortiz E, et al. Prevalence of fungal involvement in a series of patients with nail psoriasis. Clin Exp Dermatol. 2008 Mar;33(2):194-5.

5 Salomon J, Szepietowski JC, Proniewicz A. Psoriatic nails: a prospective clinical study. J Cutan Med Surg. 2003 Jul-Aug;7(4):317-21.

6 Ovcina-Kurtovic N, Kasumagic-Halilovic E. Prevalence of nail abnormalities in patients with psoriasis. Our Dermatol Online. 2013 Jun;4(3):272-4.

7 Brazzelli V, Carugno A, Alborghetti A, Grasso V, Cananzi R, Fornara L, et al. Prevalence, severity and clinical features of psoriasis in fingernails and toenails in adult patients: italian experience. J Eur Acad Dermatol Venereol. 2012 Nov;26(11):1354-9.

8 Klaassen KM, Dulak MG, van de Kerkhof PC, Pasch MC. The prevalence of onychomycosis in psoriatic patients: a systematic review. J Eur Acad Dermatol Venereol. 2014 May;28(5): $533-41$.

9 Zargari O, Leyli EK, Azimi SZ. Nail involvement in patients with psoriatic arthritis in Northern Iran. Autoimmune Dis. 2018 Oct; 2018:4608490.

10 Augustin M, Reich K, Blome C, Schäfer I, Laass A, Radtke MA. Nail psoriasis in Germany: epidemiology and burden of disease. Br J Dermatol. 2010 Sep;163(3):580-5.

11 Chen K, Wang G, Jin H, Xu J, Zhu X, Zheng $\mathrm{M}$, et al. Clinic characteristics of psoriasis in China: a nationwide survey in over 12000 patients. Oncotarget. 2017 Jul;8(28):46381-9.

12 Shemer A, Trau H, Davidovici B, Grunwald $\mathrm{MH}$, Amichai B. Onychomycosis in psoriatic patients - rationalization of systemic treatment. Mycoses. 2010 Jul;53(4):340-3.
13 Mesrati H, Amouri M, Chaari I, Bahloul E, Chaabane $\mathrm{H}$, Masmoudi A, et al. Le psoriasis unguéal: une étude prospective. Ann Dermatol Venereol. 2013 Dec;140(12 Suppl 1):4778.

14 van der Velden HM, Klaassen KM, van de Kerkhof PC, Pasch MC. Fingernail psoriasis reconsidered: a case-control study. J Am Acad Dermatol. 2013 Aug;69(2):245-52.

15 Tham SN, Lim JJ, Tay SH, Chiew YF, Chua TN, Tan E, et al. Clinical observations on nail changes in psoriasis. Ann Acad Med Singapore. 1988 Oct;17(4):482-5.

16 de Jong EM, Seegers BA, Gulinck MK, Boezeman JB, van de Kerkhof PC. Psoriasis of the nails associated with disability in a large number of patients: results of a recent interview with 1,728 patients. Dermatology. 1996; 193(4):300-3.

17 Hallaji Z, Babaeijandaghi F, Akbarzadeh M, Seyedi SZ, Barzegari M, Noormohammadpour $\mathrm{P}$, et al. A significant association exists between the severity of nail and skin involvement in psoriasis. J Am Acad Dermatol. 2012 Jan;66(1):e12-3.

18 Gupta AK, Daigle D, Foley KA. The prevalence of culture-confirmed toenail onychomycosis in at-risk patient populations. J Eur Acad Dermatol Venereol. 2015 Jun;29(6): 1039-44.

19 Rigopoulos D, Papanagiotou V, Daniel R 3rd, Piraccini BM. Onychomycosis in patients with nail psoriasis: a point to point discussion. Mycoses. 2017 Jan;60(1):6-10.

20 Carrillo-Meléndrez H, Ortega-Hernández E, Granados J, Arroyo S, Barquera R, Arenas R. Role of HLA-DR Alleles to Increase Genetic Susceptibility to Onychomycosis in Nail Psoriasis. Skin Appendage Disord. 2016 Sep;2(12):22-5.

21 Larsen GK, Haedersdal M, Svejgaard EL. The prevalence of onychomycosis in patients with psoriasis and other skin diseases. Acta Derm Venereol. 2003;83(3):206-9.

22 Palatsi R, Hägg P. [The immune response against microbial infections in the skin - weak in atopic dermatitis and strong in psoriasis]. Duodecim. 2011;127(2):127-34. Finnish.
23 Ortonne JP, Baran R, Corvest M, Schmitt C, Voisard JJ, Taieb C. Development and validation of nail psoriasis quality of life scale (NPQ10). J Eur Acad Dermatol Venereol. 2010 Jan;24(1):22-7.

24 Kaçar N, Ergin S, Ergin C, Erdogan BS, Kaleli I. The prevalence, aetiological agents and therapy of onychomycosis in patients with psoriasis: a prospective controlled trial. Clin Exp Dermatol. 2007 Jan;32(1):1-5.

25 Zisova L, Valtchev V, Sotiriou E, Gospodinov $\mathrm{D}$, Mateev G. Onychomycosis in patients with psoriasis-a multicentre study. Mycoses. 2012 Mar;55(2):143-7.

26 Romaszkiewicz A, Bykowska B, Zabłotna M, Sobjanek M, Sławińska M, Nowicki RJ. The prevalence and etiological factors of onychomycosis in psoriatic patients. Postepy Dermatol Alergol. 2018 Jun;35(3):309-13.

27 Leibovici V, Hershko K, Ingber A, Westerman M, Leviatan-Strauss N, Hochberg M. Increased prevalence of onychomycosis among psoriatic patients in Israel. Acta Derm Venereol. 2008;88(1):31-3.

28 Szepes E. Mykotische infektionen psoriatischer nägel/Mycotic infections of psoriatic nails. Mycoses. 1986 Feb;29(2):82-4.

29 Staberg B, Gammeltoft M, Onsberg P. Onychomycosis in patients with psoriasis. Acta Derm Venereol. 1983;63(5):436-8.

30 Ständer H, Ständer M, Nolting S. [Incidence of fungal involvement in nail psoriasis]. Hautarzt. 2001 May;52(5):418-22. German.

31 Hamnerius N, Berglund J, Faergemann J. Pedal dermatophyte infection in psoriasis. $\mathrm{Br}$ J Dermatol. 2004 Jun;150(6):1125-8.

32 Natarajan V, Nath AK, Thappa DM, Singh R, Verma SK. Coexistence of onychomycosis in psoriatic nails: a descriptive study. Indian J Dermatol Venereol Leprol. 2010 Nov-Dec; 76(6):723.

33 Chaowattanapanit S, Pattanaprichakul P, Leeyaphan C, Chaiwanon O, Sitthinamsuwan P, Kobwanthanakun W, et al. Coexistence of fungal infections in psoriatic nails and their correlation with severity of nail psoriasis. Indian Dermatol Online J. 2018 Sep-Oct;9(5): 314-7.
Prevalence of OM in Patients with Psoriasis
Skin Appendage Disord 2019;5:299-303 DOI: $10.1159 / 000497825$ 